\title{
Population-based cohort study on comparative effectiveness and safety of biologics in inflammatory bowel disease
}

This article was published in the following Dove Press journal:

Clinical Epidemiology

\author{
Riccardo Di \\ Domenicantonio' \\ Francesco Trotta' \\ Silvia Cascini' \\ Nera Agabiti' \\ Anna Kohn² \\ Antonio Gasbarrini ${ }^{3}$ \\ Marina Davoli' \\ Antonio Addis' \\ 'Department of Epidemiology, Lazio \\ Regional Health Service, Rome, Italy; \\ 2IBD Unit, AO San Camillo Forlanini, \\ Rome, Italy; ${ }^{3}$ Department of Internal \\ Medicine, Agostino Gemelli University \\ Hospital, Catholic University of \\ Sacred Heart, Rome, Italy
}

Correspondence Riccardo Di Domenicantonio

Department of Epidemiology, Lazio Regional Health Service, Via Cristoforo Colombo, Rome II 2-00 I47, Italy Email r.didomenicantonio@deplazio.it
Background: The comparison of effectiveness and safety of anti-tumor necrosis factor-alpha agents for the treatment of inflammatory bowel disease (IBD) is relevant for clinical practice and stakeholders.

Objective: The objective of this study was to compare the risk of abdominal surgery, steroid utilization, and hospitalization for infection in Crohn's disease (CD) or ulcerative colitis (UC) patients newly treated with infliximab (IFX) or adalimumab (ADA).

Methods: A retrospective population-based cohort study was performed using health information systems data from Lazio region, Italy. Patients with CD or UC diagnosis were enrolled at first prescription of IFX or ADA during 2008-2014 (index date). Only new drug users were followed for 2 years from the index date. IFX versus ADA adjusted hazard ratios were calculated applying "intention-to-treat" approach, controlling for several characteristics and stratifying the analysis on steroid use according to previous drug utilization. Sensitivity analyses were performed according to "as-treated" approach, adjusting for propensity score, censoring at switching or discontinuation, and evaluating different lengths of follow-up periods.

Results: We enrolled 1,432 IBD patients ( $42 \%$ and $83 \%$ exposed to IFX for CD and UC, respectively). In both diseases, treatment effects did not differ in any outcome considered, and sensitivity analyses confirmed the results from the main analysis.

Conclusion: In our population-based cohort study, effectiveness and safety data in new users of ADA or IFX with CD or UC were comparable for the outcomes we tested.

Keywords: biologics, real-world effectiveness, inflammatory bowel disease

\section{Background}

Crohn's disease (CD) and ulcerative colitis (UC) are chronic, relapsing conditions resulting from uncontrolled inflammation of the intestinal mucosa. Infliximab (IFX) and adalimumab (ADA) are biologic agents targeting tumor necrosis factor-alpha (TNF- $\alpha$ ), a key factor in the pathway of the immune response, and are approved for use in patients with moderate-to-severe inflammatory bowel disease (IBD). ${ }^{1}$ Several meta-analyses and network meta-analyses lead to an estimate of comparative efficacy between the various biologic agents, through indirect comparisons of individual anti-TNF- $\alpha$ agents relative to a common comparator. ${ }^{2-6}$ Evidence is available from clinical studies comparing single agents versus placebo, and all agents were superior with respect to induction and maintenance therapy in the treatment of CD and UC. ${ }^{7}$ Head-to-head trials between different anti-TNF- $\alpha$ agents are still warranted in order to establish the best therapeutic option. Meta-analyses of biologic agents for the treatment of IBD have not raised any important issues in terms of safety, ${ }^{5,8}$ which is 
believed to be generally comparable between the anti-TNF- $\alpha$ agents, although evidence exists concerning specific adverse events, such as melanoma, ${ }^{9}$ opportunistic infections, and lymphoma. ${ }^{10-12}$

In general, clinical trials hardly reach the statistical power to detect rare events due to limited sample size and the length of follow-up. This can be overcome by performing large population-based observational studies, taking the opportunity of routinely available data from health information systems, which allow for direct comparison between single agents in real-world clinical practice. ${ }^{3}$ Few recent studies compared the effectiveness and safety of biologic agents in IBD patients using real word-data, indicating no evidence of superiority of any agent. ${ }^{13-18}$ The aim of this study was to compare the long-term risk of bowel surgery, steroid utilization, and hospitalization for infection between new users of IFX and ADA treated for IBD.

\section{Methods}

\section{Data sources}

Data for this study were from the Lazio region health administrative databases. All Lazio region population (5.6 million) was included in the Italian National Health Service (INHS), which provides universal health insurance for its residents, with free-of-charge coverage for general practitioner and hospital services. Drugs and specialist care are also guaranteed free of charge by the INHS for several chronic diseases, provided that the diagnosis and the prescriptions are performed and certified by INHS specialists. The data of all individuals with disease-specific payment exemptions have been recorded in the disease-specific payment exemptions register (DPER) since 2005.

We conducted a retrospective population-based cohort study in the Lazio region (about 6 million inhabitants), using hospital discharges, pharmacy, death certificates, and DPER data. The hospital information system (HIS) provided information on hospitalizations, which includes patients' demographic characteristics, admission and discharge dates, up to six International Classification of Diseases, Ninth Revision, Clinical Modification (ICD-9-CM) discharge diagnoses, and up to six ICD-9-CM hospital procedures. The DPER included all individuals who benefitted from copayment exemptions for drugs or specialist services. The regional drug claims register (RDCR) comprised individual records for each medical prescription that is dispensed from public and private pharmacies and the date of dispensing, since 2006. The registry was limited to drugs dispensed to outpatients and reimbursed by the health care system. Drugs were identified by the national drug register code, which is related to the Anatomical Therapeutic Chemical (ATC) classification system. The regional population registry (PR) comprised the place of residence and dates of health insurance coverage for all enrollees of the Lazio Regional Health Service. Death certificates from the regional mortality register were used to update the PR. All data sources can be linked between them using anonymous keys. We used PR, HIS, and DPER to select the study population and track subjects' eligibility during the follow-up; RDCR to characterize the exposure; and HIS and RDCR to determine coexisting conditions and drug consumption, respectively.

\section{Population}

We selected IBD patients through an algorithm previously validated in Italy ${ }^{19}$ and classified CD or UC according to a similar approach reported by Benchimol et al. ${ }^{20}$ From HIS, we collected all hospital admissions for IBD (including CD or UC) of all residents in the Lazio region between January 1, 2002, and December 31, 2014. Enrollment was integrated by patients included in the DPER between January 1, 2006, and December 31,2014, with a copayment exemption for CD or UC (Figure 1; for details refer Tables S1 and $\underline{\mathrm{S} 2}$ ). From this population $(n=31,880)$, we selected only patients with at least one prescription for the drugs under study from January 1,2008 , to December 31, 2014. We defined the index date as the date of the first prescription of any study drug.

Exclusion criteria were as follows: not being registered in the regional health care system during the study period, diagnosis of both UC and CD in different instances (unspecified IBD), discharge with a diagnosis of other diseases with indications for anti-TNF- $\alpha$ therapy or evidence of drug therapies indicated for autoimmune disease other than IBD within the 24 months prior to the index date (for details, refer Tables S3 and S4).

\section{Exposure}

Biologic drugs under study were anti-TNF- $\alpha$ ADA (ATC code: L04AB04) and IFX (ATC code: L04AB02). Exposure was defined in terms of defined daily doses (DDDs); the number of DDDs available was translated into the number of days in which the patient was treated, counting one DDD per day and distributing all available DDDs to the days of follow-up starting on the index date and allowing also for the use of accumulated DDDs over time. Only new users of the drug were included, considering a 12-month washout period before the index date during which the patient did not use any antiTNF- $\alpha$ agent, comprising etanercept (ATC code: L04AB01). 
Patients discharged with diagnosis of CD or UC (years: 2002-2014)

or with active copayment exemption for CD or UC (years: 2006-2014) 31,880

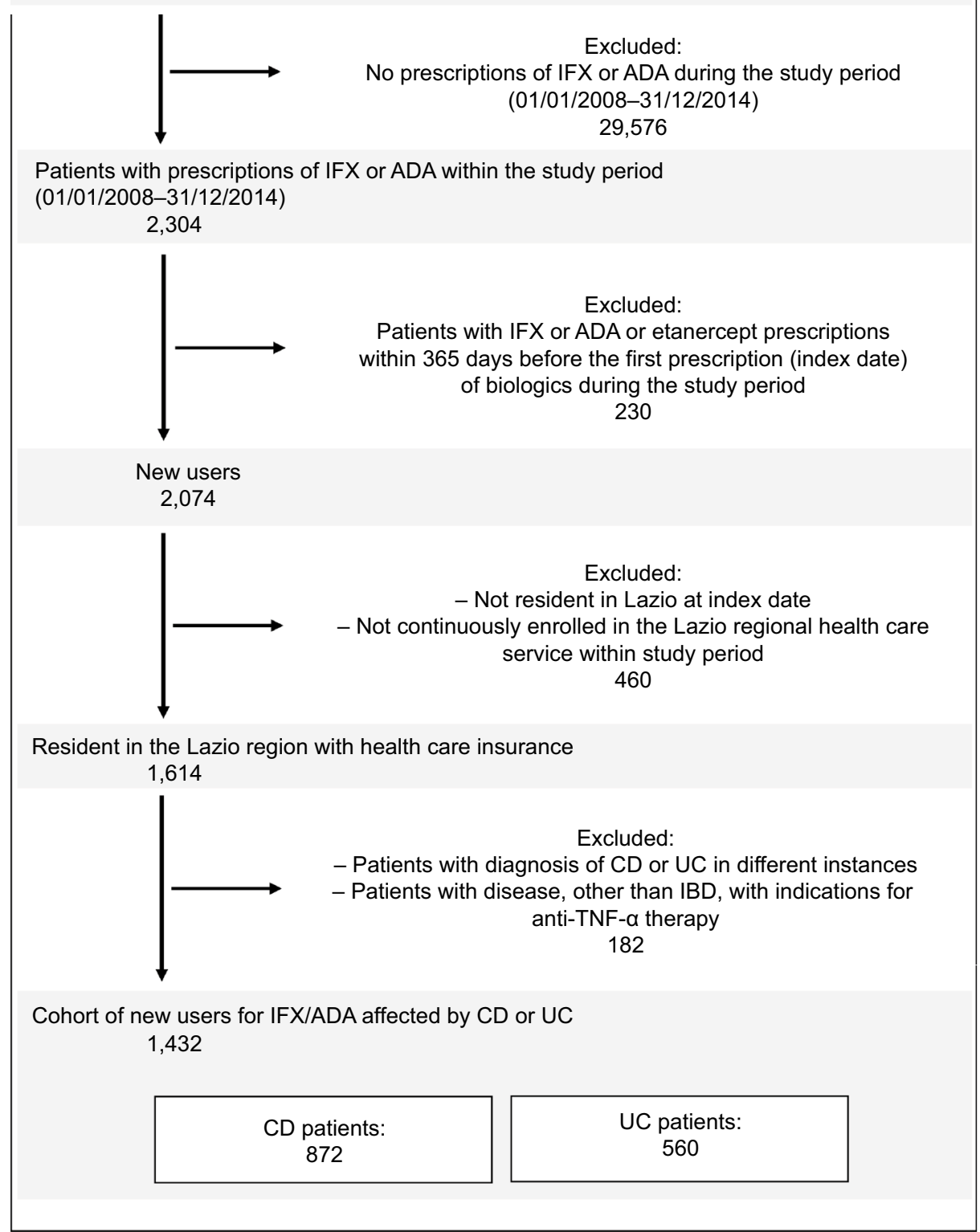

Figure I Cohort selection.

Abbreviations: ADA, adalimumab; CD, Crohn's disease; IBD, inflammatory bowel disease; IFX, infliximab; UC, ulcerative colitis.

\section{Potential confounders/effect modifiers}

Demographic characteristics and coexisting conditions were determined from PR and HIS, respectively, and drug consumption was determined from RDCR. Patient's age at index date was classified in 5 classes for $\mathrm{CD}$ and 3 classes for UC. Area of residence was categorized in three groups: the municipality of Rome, the province of Rome, and the outer Lazio provinces. The following comorbidities were retrieved from HIS within 12 months before the index date: ischemic disease, congestive heart failure, cerebrovascular diseases, dementia, peripheral vascular disease, diabetes, anxiety, neurological disease, psychiatric disease, renal disease, liver disease, and hypertension (ICD-9-CM codes in Table S5). The overall number of comorbidities served as an indicator for multicomorbidity with the following categorization: none, one condition, and two or more conditions. Similarly, the following IBD-related conditions were retrieved from hospital diagnosis to characterize the diseases: anemia or malabsorption, intestinal stenosis or obstruction, fistula/abscess/ulcer of intestine, hemorrhage, and previous abdominal surgery 
(ICD-9-CM codes in Table S6). Disease forms were defined by categorizing last discharge main diagnosis that occurred before the index date by the fourth digit of ICD-9-CM code. Disease course was classified using the time between the index date and the last evidence of IBD (from HIS or DPER) in the following two groups: within 5 years before the index date and $>5$ years before the index date. Drug utilization in the 12 months prior to the index date was considered for the following pharmacological treatments indicated for IBD: antimetabolites, immunosuppressants, antibiotics, corticosteroids, aminosalicylic acid and similar agents, and antibacterials (ATC codes in Table S7). Patients were classified with respect to the date of the last prescription in the 12 months prior to the index date as remote users ( $>6$ months), recent users ( $<6$ months), and no users (no prescriptions).

\section{Study design}

The main analytical approach used was the intention-to-treat approach (ITT), in which patients were classified into two groups according to their exposure at the index date. The patients were followed from the index date until outcome, censoring at death, or until the end of the study (2 years after the index date), whatever happened first. Furthermore, we performed a sensitivity analysis using an as-treated (AT) analysis based on censoring at switching. We classified exposure at the index date and followed patients from the index date until the occurrence of outcome, censoring at death, switching or discontinuation, or until the end of the study ( 2 years after the index date), whatever happened first. Regarding discontinuation, at the end of a period of exposure (ie, when all available DDDs were expired), a renewal grace time of 60 days was defined, during which the patient could claim the drug without being censored, still being considered exposed to the treatment. In case of switching to an alternative drug treatment, a switching grace time of 7 days was applied, considering a lagged effect of the first drug after its last intake and attributing outcomes occurring in these days to the first treatment.

\section{Outcomes}

Effectiveness outcomes considered were the following: 1) the first IBD-related bowel surgery (IBD-related procedures in Table S8); and 2) the first steroid prescription occurred at least 60 days after the start of anti-TNF- $\alpha$ therapy. Our safety outcome was the first hospital admission for opportunistic and serious infections retrieved through HIS (ICD-9-CM codes in Table S9). Finally, due to small numbers, some relevant adverse events (infusion reaction, dermatomyositis, brain neoplasm, and acquired hemolytic anemia) were only described in terms of frequencies (ICD-9-CM codes and frequencies in Table S10).

\section{Statistical analysis}

We performed unadjusted time-to-event analysis, examining Kaplan-Meier survival curves for abdominal surgery, steroid utilization, hospitalization for infection, and discontinuation or switch. Differences between ADA and IFX survival curves were evaluated by the log rank test. Outcome rates (per 100 person-years) were calculated as the number of events divided by the person-time at risk. To compare the effectiveness and safety of IFX and ADA, we performed multivariate analysis using Cox proportional hazard models, with age as a timescale. We calculated hazard ratios (HRs) and related 95\% confidence intervals (95\% CIs) for each outcome among patients treated with IFX compared with ADA. In the ITT analysis, adjusted estimates were obtained by using a stepwise Cox regression $(p$-entry $=0.05, p$-stay $=0.1)$ to select clinical characteristics associated with each outcome, separately for CD and UC patients; then, we included the exposure effect in the predictive models (see Tables S11-S13).

Furthermore, three sensitivity analyses were performed. In the first sensitivity analysis, the ITT main analysis was replicated applying an AT approach. In the AT analysis, we performed risk adjustment using quintiles of the propensity score. We calculated the propensity score to balance groups on baseline characteristics in order to reduce the impact of observed confounding. The likelihood of using IFX was estimated for $\mathrm{CD}$ patients, using the following characteristics (selected by a stepwise procedure): gender, age class, residence, CD form, time from last evidence of IBD from HIS to the index date, fistula or ulcer, or abscess of intestine. In the same manner, we estimated the likelihood of using IFX for UC patients using the following characteristics: gender, age class, residence, and UC disease form. Patients were stratified into quintiles according to their predicted propensity score, and the quintiles of the propensity score were used as a covariate together with the treatment variable to be fitted in the Cox proportional model. This method assumes that treated and control patients with the same quintile of the propensity score have the same distribution of measured baseline characteristics ${ }^{21}$ (Tables S14 and S15).

In order to evaluate the results according to shorter follow-up periods, we performed a second sensitivity analysis, restricted to steroid utilization considering a follow-up period of only 1 year through an AT study design. The third sensitivity analysis focused on CD patients after the exclu- 
sion of abdominal surgeries occurred within 60 days from the index date aiming at minimizing the misclassification of nonresponders and confounding by disease severity.

Proportionality of hazards assumption was met for all outcomes, with the exception of the steroid prescription outcome in the ITT study design; thus, we stratified this analysis according to previous corticosteroid utilization considering the following two categories: recent users and remote or no users. All statistical analyses were performed by using SAS Version 9.2 (SAS institute, Cary, NC, USA).

\section{Ethics statement}

The Department of Epidemiology of the Lazio Regional Health Service is the regional referral center for epidemiological research and has full access to anonymized health information systems. Data were routinely collected by the health information system from the Regional Department of the Lazio Regional Health Service, which anonymized all the records prior to the analysis performed by our Department of Epidemiology of the Lazio Regional Health Service. The authors did not anonymize the data. Ethics committee or institutional review board approval and informed consent were not necessary because the authors used the data already collected at the beginning of the study, and the data were analyzed anonymously through a standardized methodology according to the Italian national privacy law (national legislative decree on privacy policy no. 196/30 June 2003). The linkage method used a unique anonymous patient identifier deriving from information on persons' names, date and place of birth, and gender, according to Italian privacy legislation. Individuals cannot be identified directly or through identifiers, and the results are shown in aggregate form.

\section{Results}

\section{Cohort selection and patient characteristics}

We identified 2,304 patients exposed to the study drugs, 2,074 of whom were classified as new users. After the application of exclusion criteria, the cohort consisted of 1,432 patients, 872 patients with $\mathrm{CD}$ and 560 patients with UC (Figure 1). Tables 1 and 2 describe the demographic, clinical, and drug utilization characteristics of patients according to the treatment group. Among $\mathrm{CD}$ and UC patients, respectively, $367(42.1 \%)$ and $469(83.7 \%)$ were classified as IFX new users. The median age of the population was 41 (range $=7-82$ ) years. For both diseases, we observed a similar pattern of association between patient characteristics and the two study drugs. IFX users were more likely to be male, had more recently been discharged from hospital, or obtained an exemption for their condition. They were affected by a more extended disease form (555.2 regional enteritis of small intestine with large intestine for $\mathrm{CD}$ and 556.6 universal UC for UC), and their condition was more severe (intestinal fistula or ulcer/abscess in CD and anemia/ hemorrhage in UC). Drug utilization patterns were comparable between ADA and IFX users in both diseases, except for aminosalicylic acid and corticosteroids in UC patients, which showed a higher consumption among IFX users. Tables S14 and S15 report the characteristics of patients and the expected probability of being treated with each therapy (adjusted for all characteristics measured at baseline) used in the propensity score.

\section{Unadjusted survival analysis}

Figure 2 reports, for each outcome, the Kaplan-Meier survival curves for the ITT analysis. Table S16 report the results for abdominal surgery and infections among UC patients. We observed no differences in unadjusted time-to-event analysis in all outcomes considered. The results of AT analysis showed that switch and discontinuation were more frequent among ADA users compared with IFX users in both diseases (log rank test $\operatorname{pr}>\chi^{2} \leq 0.001$; Figure S1).

\section{Rates, crude, and adjusted HRs}

Among CD patients, the overall incidence rates (per 100 person-years) of abdominal surgery and hospitalization for infections were 6.84 and 1.03, respectively. Among UC patients, we observed rates of 6.05 for abdominal surgery and 1.14 for hospitalization for infections. Incidence rates for steroid utilization after the index date were 55.6 among recent users and 20.9 for remote or no users in CD and 86.9 and 34.9 for UC, respectively.

In Table 3, rates and HRs of abdominal surgery, hospitalization for infection, and steroid utilization among patients treated with IFX versus those treated with ADA were reported. Table S16 reports the results for abdominal surgery and infections outcomes. For CD, the adjusted HRs of abdominal surgery and hospitalization for infections did not indicate differences between treatments. Similarly, the results of the steroid utilization analysis, stratified by previous drug utilization, did not highlight differences among agents for both diseases, with adjusted $\mathrm{HR}$ of $1.16(95 \% \mathrm{CI}=0.92-1.46)$ and $1.01(95 \% \mathrm{CI}=0.70-1.47)$, respectively, for recent users and remote or no users in $\mathrm{CD}$ and $1.21(95 \% \mathrm{CI}=0.87-1.68)$ and $0.67(95 \% \mathrm{CI}=0.35-1.28)$ in $\mathrm{UC}$, respectively. 
Table I Cohort characteristics according to drug exposure. Crohn's disease

\begin{tabular}{|c|c|c|c|}
\hline Characteristic & $\begin{array}{l}\text { Infliximab (\%) } \\
n=367\end{array}$ & $\begin{array}{l}\text { Adalimumab (\%) } \\
n=505\end{array}$ & $p$-value \\
\hline \multicolumn{4}{|l|}{ Demographic } \\
\hline \multicolumn{4}{|l|}{ Gender } \\
\hline Female & 44.7 & 54.1 & 0.006 \\
\hline \multicolumn{4}{|l|}{ Age-group (years) } \\
\hline $0-19$ & 10.0 & 6.5 & 0.26 \\
\hline $20-34$ & 28.3 & 30.3 & \\
\hline $35-49$ & 31.9 & 34.1 & \\
\hline $50-64$ & 24.3 & 22.0 & \\
\hline$>64$ & 5.5 & 7.1 & \\
\hline \multicolumn{4}{|l|}{ Disease characteristics } \\
\hline \multicolumn{4}{|l|}{ Time from the index date to last evidence of IBD (from HIS or DPER) } \\
\hline More than 5 years before the index date & 37.3 & 45.1 & 0.021 \\
\hline \multicolumn{4}{|l|}{ Disease form (main diagnosis in the last discharge before index date) } \\
\hline 555.0 Regional enteritis of small intestine & 23.7 & 28.7 & $<0.001$ \\
\hline 555.I Regional enteritis of large intestine & 10.4 & 6.5 & \\
\hline 555.2 Regional enteritis of small intestine with large intestine & 32.2 & 25.9 & \\
\hline 555.9 Regional enteritis of unspecified site & 18.8 & 21.8 & \\
\hline UC diagnosis in CD patients & 4.9 & 1.4 & \\
\hline Patients not discharged within 5 years before the index date & 10.1 & 15.6 & \\
\hline \multicolumn{4}{|l|}{ Comorbidities/risk factor } \\
\hline Anemia/hemorrhage & 10.9 & 7.3 & 0.066 \\
\hline Intestinal fistula or ulcer/abscess & 26.2 & 13.1 & $<0.001$ \\
\hline Intestinal obstruction/stenosis & 6.8 & 6.3 & 0.78 \\
\hline Previous surgery & 12.0 & 14.5 & 0.29 \\
\hline \multicolumn{4}{|l|}{ Number of comorbidities } \\
\hline 1 & 7.6 & 5.5 & 0.46 \\
\hline 2 or more & 1.9 & 1.8 & \\
\hline \multicolumn{4}{|l|}{ Drug utilization } \\
\hline \multicolumn{4}{|l|}{ Aminosalicylic acid } \\
\hline Recent & 61.6 & 57.0 & 0.145 \\
\hline Remote & 10.9 & 9.3 & \\
\hline \multicolumn{4}{|l|}{ Immunosuppressant } \\
\hline Recent & 25.6 & 26.7 & 0.93 \\
\hline Remote & 4.9 & 5.0 & \\
\hline \multicolumn{4}{|l|}{ Antimetabolites } \\
\hline Recent & 9.3 & 8.1 & 0.151 \\
\hline Remote & I.I & 3.0 & \\
\hline \multicolumn{4}{|l|}{ Steroids (local/systemic) } \\
\hline Recent & 56.4 & 58.2 & 0.33 \\
\hline Remote & 9.8 & 12.1 & \\
\hline \multicolumn{4}{|l|}{ Antibacterials } \\
\hline Recent & 60.2 & 53.3 & 0.075 \\
\hline Remote & 13.1 & 13.1 & \\
\hline
\end{tabular}

Notes: Recent, within 6 months from the index date; remote, within $>6$ months from the index date.

Abbreviations: CD, Crohn's disease; DPER, disease-specific payment exemptions register; HIS, hospital information system; IBD, inflammatory bowel disease; UC, ulcerative colitis.

\section{Sensitivity analysis}

Sensitivity analysis conducted applying the AT study design confirmed the results shown in the ITT analysis for abdominal surgery and hospitalization for infection outcomes (data not reported). Table 4 reports, for steroid utilization, the results of the first sensitivity analysis; the results of the application of a follow-up period of only 1 year are also reported (second sensitivity analysis). Differently from main analysis, we found an effect favoring ADA with adjusted HRs of 1.49 (95\% CI $=1.11-2.00)$ among UC patients and, applying a shorter follow-up, of $1.36(95 \% \mathrm{CI}=1.06-1.73)$ among CD patients. 
Table 2 Cohort characteristics according to drug exposure. Ulcerative colitis

\begin{tabular}{|c|c|c|c|}
\hline Characteristic & $\begin{array}{l}\text { Infliximab (\%) } \\
\mathrm{n}=469\end{array}$ & $\begin{array}{l}\text { Adalimumab (\%) } \\
\mathrm{n}=91\end{array}$ & $p$-value \\
\hline \multicolumn{4}{|l|}{ Demographic } \\
\hline \multicolumn{4}{|l|}{ Gender } \\
\hline Female & 42.0 & 56.7 & 0.010 \\
\hline \multicolumn{4}{|l|}{ Age-group (years) } \\
\hline $0-34$ & 33.6 & 33.3 & 0.70 \\
\hline $35-49$ & 33.8 & 30.0 & \\
\hline$>49$ & 32.6 & 36.7 & \\
\hline \multicolumn{4}{|l|}{ Disease characteristics } \\
\hline \multicolumn{4}{|l|}{ Time from index date to last evidence of IBD (from HIS or DPER) } \\
\hline More than 5 years before index date & 32.4 & 46.2 & 0.010 \\
\hline \multicolumn{4}{|l|}{ Disease form (main diagnosis in the last discharge before index date) } \\
\hline 556.0 Ulcerative enterocolitis/556.I ulcerative ileocolitis/556.4 pseudopolyposis & 7.7 & 5.5 & 0.000 \\
\hline 556.2 Ulcerative proctitis/556.3 ulcerative proctosigmoiditis & 10.9 & 7.7 & \\
\hline 556.5 Left-sided ulcerative colitis & 14.1 & 4.4 & \\
\hline 556.6 Pancolitis & 29.4 & 12.1 & \\
\hline 556.8 Other ulcerative colitis/556.9 ulcerative colitis, unspecified & 15.4 & 15.4 & \\
\hline $\mathrm{CD}$ diagnosis in UC patients & 7.0 & 16.5 & \\
\hline Patients not discharged within 5 years before the index date & 15.6 & 38.5 & \\
\hline \multicolumn{4}{|l|}{ Comorbidities/risk factor } \\
\hline Anemia/hemorrhage & 14.9 & 2.2 & 0.001 \\
\hline Intestinal fistula or ulcer/abscess & 6.6 & 4.4 & 0.43 \\
\hline Intestinal obstruction/stenosis & 1.3 & - & \\
\hline Previous surgery & 2.1 & 2.2 & 0.96 \\
\hline \multicolumn{4}{|l|}{ Number of comorbidities } \\
\hline I & 11.9 & 8.8 & 0.183 \\
\hline 2 or more & 4.7 & 1.1 & \\
\hline None & 83.4 & 90.1 & \\
\hline \multicolumn{4}{|l|}{ Drug utilization } \\
\hline \multicolumn{4}{|l|}{ Aminosalicylic acid } \\
\hline Recent & 88.7 & 75.8 & $0.00 \mathrm{I}$ \\
\hline Remote & 3.2 & 3.3 & \\
\hline \multicolumn{4}{|l|}{ Immunosuppressant } \\
\hline Recent & 29.0 & 23.1 & 0.37 \\
\hline Remote & 6.2 & 5.5 & \\
\hline \multicolumn{4}{|l|}{ Antimetabolites } \\
\hline Recent & 6.2 & 8.8 & 0.095 \\
\hline Remote & 0.9 & 3.3 & \\
\hline \multicolumn{4}{|l|}{ Steroids (local/systemic) } \\
\hline Recent & 83.4 & 65.9 & $<0.001$ \\
\hline Remote & 6.4 & 6.6 & \\
\hline \multicolumn{4}{|l|}{ Antibacterials } \\
\hline Recent & 43.9 & 37.4 & 0.27 \\
\hline Remote & 13.0 & 19.8 & \\
\hline
\end{tabular}

Notes: Recent, within 6 months from the index date; remote, within $>6$ months from the index date.

Abbreviations: CD, Crohn's disease; DPER, disease-specific payment exemptions register; HIS, hospital information system; IBD, inflammatory bowel disease; UC, ulcerative colitis.

Regarding the third sensitivity analysis, the exclusion of the events occurred during the first 60 days from the index data did not influence the result (data not reported).

\section{Adverse events}

The occurrence of adverse events (infusion reaction, dermatomyositis, brain neoplasm, and acquired hemolytic anemia) was very low or absent in our cohort of patients (frequencies in Table S10).

\section{Discussion}

This study aimed at obtaining real-world comparative effectiveness and safety of IFX versus ADA from a regional population of IBD patients in Italy, new users of anti-TNF- $\alpha$. 

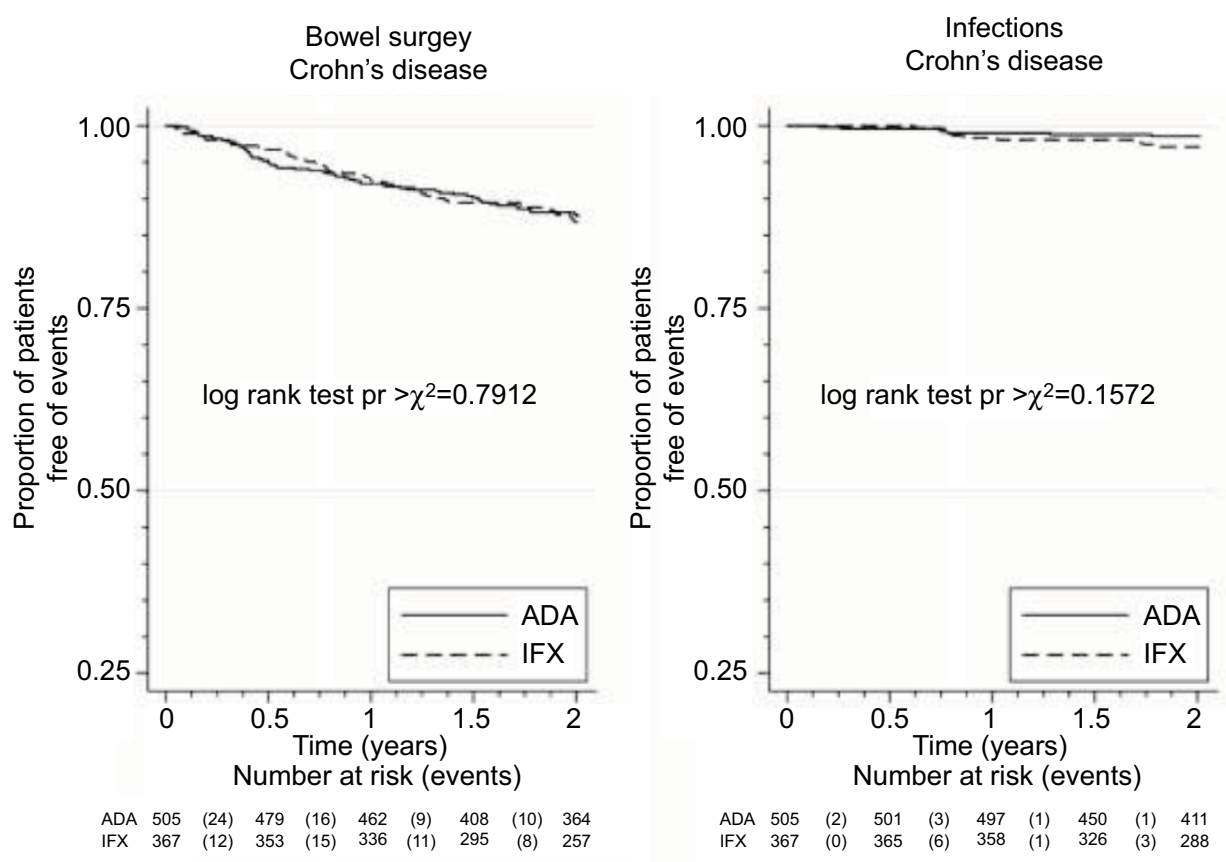

Steroid utilization (60 days after index date)

Steroid (1 year before index date)
-1 remote or no users ADA $\quad---2$ remote or no users IFX $\quad \ldots \ldots 3$ recent users ADA _ $\quad-4$ recent users IFX

Crohn's disease

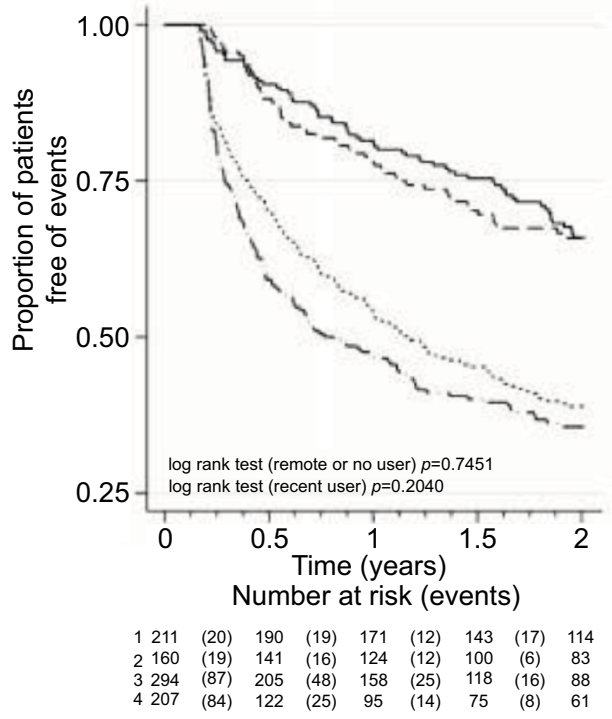

Ulcerative colitis

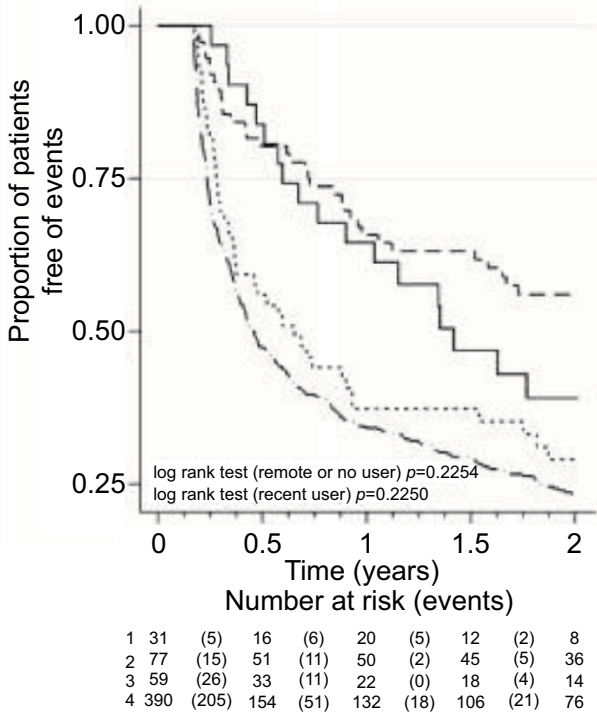

Figure 2 Intention-to-treat study design: unadjusted time-to-event analysis, Kaplan-Meier survivor curves for abdominal surgery, infections, and steroid utilization (60 days after the index date) in Crohn's disease and ulcerative colitis patients.

Abbreviations: ADA, adalimumab; IFX, infliximab.

The main finding of this study is that the HR of abdominal surgery or serious infections requiring hospitalization did not differ between IFX and ADA new users; the same was observed for steroid prescription. The first result is consistent with those of studies published in the recent years on the comparison of real-world outcomes of ADA and IFX, in IBD patients newly exposed to anti-TNF- $\alpha \cdot .^{13-18}$ Liu et $a l^{16}$ analyzed the clinical outcomes of a propensity-matched cohort of 1,030 CD patients with a median follow-up of 18 months through an ITT study design, reporting no differences between the 

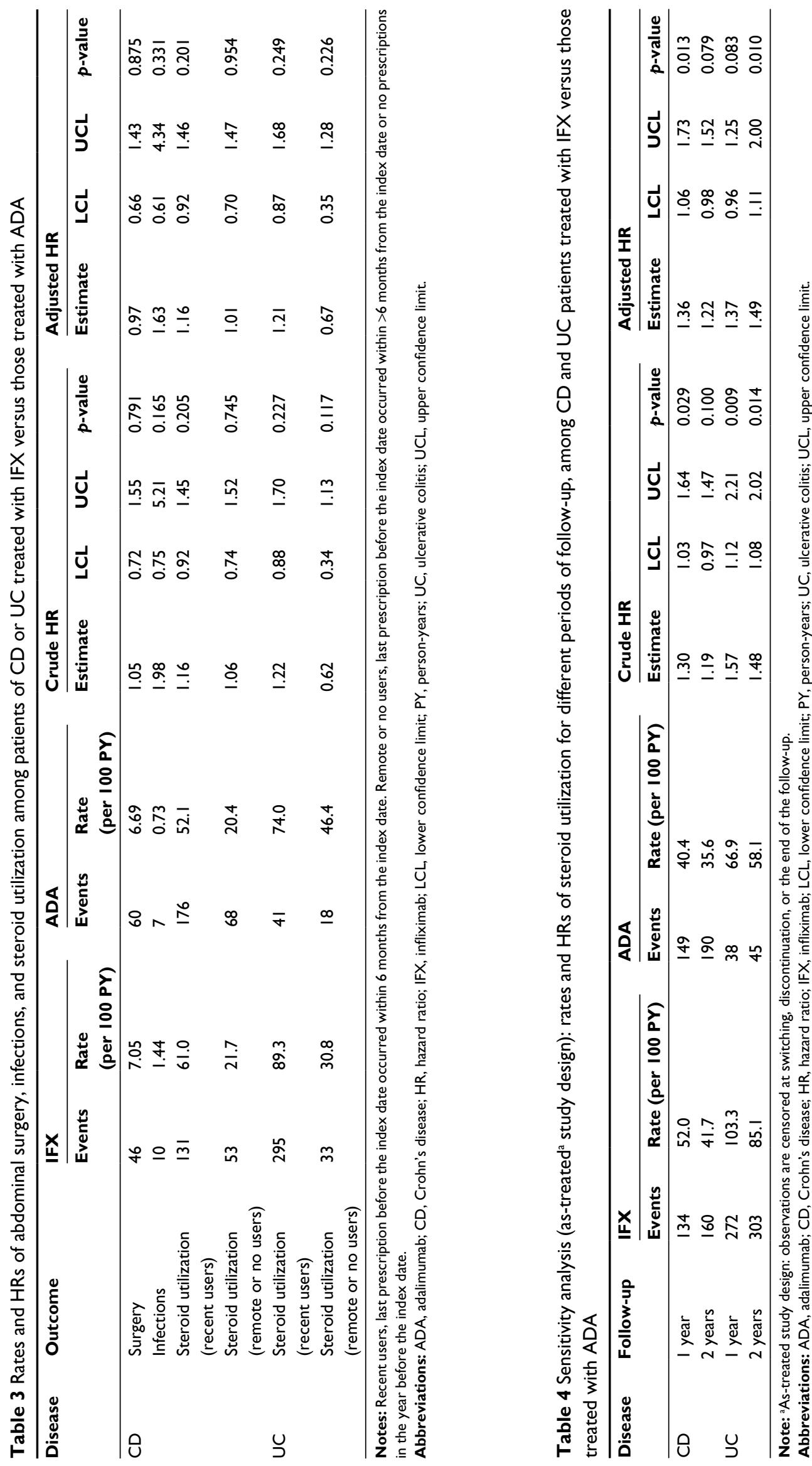
two agents in CD-specific complications and symptoms. In the same study, the rates of infections and cancers were comparable between IFX and ADA. ${ }^{16}$ Singh et al found statistically significant differences favoring IFX over ADA with respect to 1-year abdominal surgery and steroid utilization in CD patients. ${ }^{13}$ Such effects completely disappeared when 2-year and 3-year baseline cohorts were analyzed. In the same study, rates of infections were comparable between IFX and ADA. Osterman et al reported no difference in the incidence of abdominal surgery between the two agents in CD patients, although a higher risk was reported for ADA among patients aged $<65$ years. ${ }^{18}$

Regarding UC, Gies et al ${ }^{14}$ compared response rates for anti-TNF agents in "real-life" clinical practice through singlecenter cohort's data, reporting no difference in response rate. Sandborn et al, investigating a cohort of UC patients exposed to ADA ( $n=380)$ and IFX $(n=434)$, found no significant differences in time to remission, no rectal bleeding, normal stool count, and normal physician global assessment in unadjusted and adjusted comparisons. ${ }^{15}$

This is the first study to compare the effectiveness and safety of IFX and ADA in both CD and UC patients in the same population-based cohort study in Europe. We used validated algorithms to select IBD patients and to distinguish the two main forms of disease, UC and CD. ${ }^{19,20}$ Randomized controlled trials (RCTs) showed both the agents to be efficacious in CD and UC. The results from meta-analyses of RCTs suggest that no individual TNF- $\alpha$ inhibitor offers clear advantage over the others. ${ }^{2-6}$ Comparative effectiveness research (CER), conversely from RCTs, assesses how well a particular option works in a large, heterogeneous patient group treated in a real-world setting over a longer time line allowing oneto-one drug comparisons. ${ }^{22}$ Our comparative effectiveness and safety results provided complete evidence from clinical trials and network meta-analyses and also provided important information on the subpopulation of anti-TNF- $\alpha$ new users. TNF- $\alpha$ is the most important cytokine that mediates intestinal tract inflammation. ${ }^{23,24}$ Research on TNF- $\alpha$ has uncovered many pathways by which TNF- $\alpha$ may be involved in the pathogenesis of IBD; moreover, the TNF- $\alpha$ inhibitors IFX and ADA differ in their dosing regimens, pharmacokinetic properties and immunogenicity, both aspects that may affect their effectiveness and safety. ${ }^{25}$

In the ITT sensitivity analysis, after stratification by previous steroid exposure, we did not observe a difference in steroid utilization. Anyhow, Kaplan-Meier survival curves, reported in Figure 2, showed a marked reduction in the risk of steroid utilization in these patients regardless of exposure.
Moreover, previous drug utilization patterns (ie, immunomodulatory agents) resulted in markedly unbalanced result between ADA and IFX patients (data not reported). These aspects give important information to clinicians regarding the choice of treatment for IBD patients.

The different rates of switch or discontinuation between the two biologics observed may be a marker of lack of effectiveness or intolerance and patient/physician practice preferences $^{26}$ and might be useful to take into account when prescribing a biologic drug in IBD. In the main analysis, we did not find any difference in effectiveness between agents, whereas, applying the AT study design, we found among UC patients a higher risk of steroid prescription in subjects exposed to IFX. Although a deeper analysis is needed to evaluate this phenomenon, the highest rate of switch or discontinuation in ADA-treated patients could have played a role in these results.

Sensitivity analysis focused only on the first year after starting biologic therapy indicated, among CD patients, a difference in effectiveness undetected in the analysis with longer follow up. Although all published post marketing studies on these treatments do not go beyond two years of follow up, this specific aspect might suggest an extended follow-up in future CER on IBD anti TNF alpha treatments.

This study was subject to the limitations of retrospective studies based on administrative data, eg, the lack of information regarding individual dose regimens or the utilization of common strategies for improving effectiveness with biologics (dose escalation or interval shortening). Misclassification of patient's diagnosis and comorbidities, treatments, and prescriptions in administrative data cannot be excluded. Nonetheless, the coding process of such data involves several health operators (prescribers, hospital pharmacist, hospital administrator, and payer at regional level) sharing quality assurance methodologies to reduce the risk of misclassification associated with the collection of such information. Furthermore, we cannot exclude residual confounding of factors not measured in our database, such as smoking status and socioeconomic status. We acknowledge that sample size and exposure distribution were suboptimal for UC analysis, and further evidence is needed to foster the results of our study. In particular, regarding abdominal surgery and infection, the effect estimates reported for UC analysis need to be considered with caution.

\section{Conclusion}

We compared the effectiveness and safety of biologic agents between patients with IBD newly treated with IFX or ADA. 
Both agents showed a similar benefit-risk profile for the two most relevant outcomes we tested (abdominal surgery and infections).

\section{Acknowledgments}

The authors would like to thank Ursula Kirchmayer for her comments and work on editing and preparing the manuscript.

\section{Disclosure}

The authors report no conflicts of interest in this work.

\section{References}

1. D'Haens GR, Panaccione R, Higgins PD, et al. The London Position Statement of the World Congress of Gastroenterology on Biological Therapy for IBD with the European Crohn's and Colitis Organization: when to start, when to stop, which drug to choose, and how to predict response? Am J Gastroenterol. 2011;106(2):199-212; quiz 213.

2. Hazlewood GS, Rezaie A, Borman M, et al. Comparative effectiveness of immunosuppressants and biologics for inducing and maintaining remission in Crohn's disease: a network meta-analysis. Gastroenterology. 2015;148(2):344-354.e5; quiz e14-e15.

3. Stidham RW, Lee TC, Higgins PD, et al. Systematic review with network meta-analysis: the efficacy of anti-TNF agents for the treatment of Crohn's disease. Aliment Pharmacol Ther. 2014;39(12):1349-1362.

4. Singh S, Garg SK, Pardi DS, Wang Z, Murad MH, Loftus EV Jr. Comparative efficacy of biologic therapy in biologic-naive patients with Crohn disease: a systematic review and network meta-analysis. Mayo Clin Proc. 2014;89(12):1621-1635.

5. Danese S, Fiorino G, Peyrin-Biroulet L, et al. Biological agents for moderately to severely active ulcerative colitis: a systematic review and network meta-analysis. Ann Intern Med. 2014;160(10):704-711.

6. Stidham RW, Lee TC, Higgins PD, et al. Systematic review with network meta-analysis: the efficacy of anti-tumour necrosis factor-alpha agents for the treatment of ulcerative colitis. Aliment Pharmacol Ther. 2014;39(7):660-671

7. Danese S, Vuitton L, Peyrin-Biroulet L. Biologic agents for IBD: practical insights. Nat Rev Gastroenterol Hepatol. 2015;12(9):537-545.

8. Zhang D, Xiong B, Li X, Xu T, Yu M. Meta-analysis: serious adverse events in Crohn's disease patients treated with TNF-alpha inhibitors. Hepatogastroenterology. 2013;60(126):1333-1342.

9. Singh S, Nagpal SJ, Murad MH, et al. Inflammatory bowel disease is associated with an increased risk of melanoma: a systematic review and meta-analysis. Clin Gastroenterol Hepatol. 2014;12(2):210-218.

10. Ford AC, Peyrin-Biroulet L. Opportunistic infections with antitumor necrosis factor-alpha therapy in inflammatory bowel disease: meta-analysis of randomized controlled trials. Am J Gastroenterol. 2013;108(8):1268-1276.
11. Siegel CA, Marden SM, Persing SM, Larson RJ, Sands BE. Risk of lymphoma associated with combination anti-tumor necrosis factor and immunomodulator therapy for the treatment of Crohn's disease: a metaanalysis. Clin Gastroenterol Hepatol. 2009;7(8):874-881.

12. Williams CJ, Peyrin-Biroulet L, Ford AC. Systematic review with meta-analysis: malignancies with anti-tumour necrosis factor-alpha therapy in inflammatory bowel disease. Aliment Pharmacol Ther. 2014;39(5):447-458.

13. Singh S, Heien HC, Sangaralingham LR, et al. Comparative effectiveness and safety of anti-tumor necrosis factor agents in biologicnaive patients with Crohn's disease. Clin Gastroenterol Hepatol. 2016;14(8):1120-1129.e6

14. Gies N, Kroeker KI, Wong K, Fedorak RN. Treatment of ulcerative colitis with adalimumab or infliximab: long-term follow-up of a singlecentre cohort. Aliment Pharmacol Ther. 2010;32(4):522-528.

15. Sandborn WJ, Sakuraba A, Wang A, et al. Comparison of real-world outcomes of adalimumab and infliximab for patients with ulcerative colitis in the United States. Curr Med Res Opin. 2016;32(7): $1233-1241$.

16. Liu J, Sylwestrzak G, Ruggieri AP, DeVries A. Intravenous versus subcutaneous anti-TNF-alpha agents for Crohn's disease: a comparison of effectiveness and safety. J Manag Care Spec Pharm. 2015;21(7):559-566.

17. Ananthakrishnan AN, Cagan A, Cai T, et al. Comparative effectiveness of Infliximab and Adalimumab in Crohn's disease and ulcerative colitis. Inflamm Bowel Dis. 2016;22(4):880-885.

18. Osterman MT, Haynes K, Delzell E, et al. Comparative effectiveness of infliximab and adalimumab for Crohn's disease. Clin Gastroenterol Hepatol. 2014;12(5):811-817.e3.

19. Di Domenicantonio R, Cappai G, Arca M, et al. Occurrence of inflammatory bowel disease in central Italy: a study based on health information systems. Dig Liver Dis. 2014;46(9):777-782.

20. Benchimol EI, Guttmann A, Mack DR, et al. Validation of international algorithms to identify adults with inflammatory bowel disease in health administrative data from Ontario, Canada. J Clin Epidemiol. 2014;67(8):887-896.

21. Weitzen S, Lapane KL, Toledano AY, Hume AL, Mor V. Principles for modeling propensity scores in medical research: a systematic literature review. Pharmacoepidemiol Drug Saf. 2004;13(12):841-853.

22. Salleron J, Danese S, D'Agay L, Peyrin-Biroulet L. Effectiveness research in inflammatory bowel disease: a necessity and a methodological challenge. J Crohns Colitis. 2016;10(9):1096-1102.

23. Gisbert JP, Marin AC, McNicholl AG, Chaparro M. Systematic review with meta-analysis: the efficacy of a second anti-TNF in patients with inflammatory bowel disease whose previous anti-TNF treatment has failed. Aliment Pharmacol Ther. 2015;41(7):613-623.

24. Park SC, Jeen YT. Current and emerging biologics for ulcerative colitis. Gut Liver. 2015;9(1):18-27.

25. Petkau JM, Eksteen B. Selective biologics for ulcerative colitis and Crohn's disease - clinical utility of vedolizumab. Biologics. 2016;10:33-52.

26. Targownik LE, Tennakoon A, Leung S, et al. Factors associated with discontinuation of anti-TNF inhibitors among persons with IBD: a population-based analysis. Inflamm Bowel Dis. 2017;23(3):409-420.
Clinical Epidemiology

\section{Publish your work in this journal}

Clinical Epidemiology is an international, peer-reviewed, open access, online journal focusing on disease and drug epidemiology, identification of risk factors and screening procedures to develop optimal preventative initiatives and programs. Specific topics include: diagnosis, prognosis, treatment, screening, prevention, risk factor modification,

Submit your manuscript here: https://www.dovepress.com/clinical-epidemiology-journal systematic reviews, risk and safety of medical interventions, epidemiology and biostatistical methods, and evaluation of guidelines, translational medicine, health policies and economic evaluations. The manuscript management system is completely online and includes a very quick and fair peer-review system, which is all easy to use. 